This article has been accepted for publication in Monthly Notices of the Royal Astronomical Society. (C: 2020 The Authors. Published by Oxford University Press on behalf of the Royal Astronomical Society. All rights reserved. 


\title{
Spectropolarimetric observations of the CIZA J2242.8+5301 northern radio relic: no evidence of high-frequency steepening
}

\author{
F. Loi ${ }^{\oplus},{ }^{1 \star}$ M. Murgia ${ }^{\circledR},{ }^{1}$ V. Vacca ${ }^{\circledR},{ }_{1}^{1}$ F. Govoni, ${ }^{1}$ A. Melis, ${ }^{1}$ D. Wittor, ${ }^{2,4,5}$ R. Beck, ${ }^{3}$ M. Kierdorf, ${ }^{3}$ \\ A. Bonafede, ${ }^{4,5}$ W. Boschin, ${ }^{6,7,8}$ M. Brienza, ${ }^{4,5}$ E. Carretti ${ }^{\circledR},{ }^{5}$ R. Concu, ${ }^{1}$ L. Feretti, ${ }^{5}$ F. Gastaldello ${ }^{(0)}$, \\ R. Paladino, ${ }^{5}$ K. Rajpurohit ${ }^{\circledR},{ }^{4}$ P. Serra ${ }^{1}$ and F. Vazza ${ }^{\circledR 2,4,5}$ \\ ${ }^{1}$ INAF - Osservatorio Astronomico di Cagliari, Via della Scienza 5, I-09047 Selargius, Italy \\ ${ }^{2}$ University of Hamburg, Hamburger Sternwarte, Gojenbergsweg 112, D-21029 Hamburg, Germany \\ ${ }^{3}$ Max-Planck-Institut für Radioastronomie, Auf dem Hügel 69, D-53121 Bonn, Germany \\ ${ }^{4}$ Dipartimento di Fisica e Astronomia, Università di Bologna, via P. Gobetti 93/2, I-40129, Bologna, Italy \\ ${ }^{5}$ INAF - Istituto di Radioastronomia, via P. Gobetti 101, I-40129 Bologna, Italy \\ ${ }^{6}$ Fundación G. Galilei - INAF (Telescopio Nazionale Galileo), Rambla J. A. Fernández Pérez 7, E-38712 Breña Baja (La Palma), Spain \\ ${ }^{7}$ Instituto de Astrofísica de Canarias, C/Vía Láctea s/n, E-38205 La Laguna (Tenerife), Spain \\ ${ }^{8}$ Departamento de Astrofísica, Univ. de La Laguna, Av. del Astrofísico Francisco Sánchez s/n, E-38205 La Laguna (Tenerife), Spain \\ ${ }^{9}$ INAF - IASF Milano, Via Corti 12, I-20133 Milano, Italy
}

Accepted 2020 July 27. Received 2020 July 27; in original form 2020 June 4

\begin{abstract}
Observations of radio relics at very high frequency (>10 GHz) can help to understand how particles age and are (re-)accelerated in galaxy cluster outskirts and how magnetic fields are amplified in these environments. In this work, we present new single-dish 18.6 GHz Sardinia Radio Telescope and 14.25 GHz Effelsberg observations of the well-known northern radio relic of CIZA $\mathrm{J} 2242.8+5301$. We detected the relic which shows a length of $\sim 1.8 \mathrm{Mpc}$ and a flux density equal to $S_{14.25 \mathrm{GHz}}=(9.5 \pm 3.9) \mathrm{mJy}$ and $S_{18.6 \mathrm{GHz}}=(7.67 \pm 0.90) \mathrm{mJy}$ at 14.25 and $18.6 \mathrm{GHz}$, respectively. The resulting best-fitting model of the relic spectrum from $145 \mathrm{MHz}$ to $18.6 \mathrm{GHz}$ is a power-law spectrum with spectral index $\alpha=1.12 \pm 0.03$ : no evidence of steepening has been found in the new data presented in this work. For the first time, polarization properties have been derived at $18.6 \mathrm{GHz}$, revealing an averaged polarization fraction of $\sim 40$ per cent and a magnetic field aligned with the 'filaments' or 'sheets' of the relic.
\end{abstract}

Key words: polarization-galaxies: clusters: individual: CIZA J2242.8+5301 - radio continuum: general.

\section{INTRODUCTION}

The (re-)acceleration mechanism of relativistic particles in the intracluster medium (ICM) of galaxy clusters is still poorly known as well as the origin of large-scale magnetic fields in such environments.

One of the spectacular manifestations of these components is represented by diffuse radio sources, known as radio haloes and relics, respectively hosted at the centre and in the outskirts of galaxy clusters. These sources are faint $\left(S_{v} \simeq 0.1-1 \mu \mathrm{Jy} \operatorname{arcsec}^{-2}\right.$ at $1.4 \mathrm{GHz}$ ) synchrotron sources, typically with steep power-law radio spectra $\left(S_{v} \sim v^{-\alpha}\right.$, with $\left.\alpha \sim 1\right)$, which extend over Mpc-scales (see van Weeren et al. 2019, for a recent review). Radio relics are usually associated with shock waves propagating in the ICM as a consequence of galaxy cluster merging phenomena. This coincidence (Ensslin et al. 1998) supports the diffusive shock acceleration (DSA, Drury 1983; Blandford \& Ostriker 1978) as a mechanism of reacceleration of cosmic ray particles up to the $\sim \mathrm{GeV}$ energies required to explain the observed emission. According to DSA, particles scatter from magnetic field inhomogeneities and they cross the shock wave back and forth gaining energy at each crossing. The DSA mechanism

^E-mail: francesca.loi@inaf.it generates a power-law energy distribution and, if the cooling of the particles is balanced by the injection of relativistic particles, a powerlaw behaviour of the flux density as a function of frequency. This trend has been observed in a large number of radio relics suggesting that these sources are produced by the DSA mechanism. Other observed characteristics of radio relics are a gradual spectral index steepening toward the cluster centre, indicating that particles are ageing in the post-shock region, and a high degree of polarization across the relic which suggests that the magnetic field has been compressed in a thin layer. Indeed, shock compression can amplify the magnetic field component perpendicular to the shock direction. According to the models, the different mechanisms of magnetic field amplification can result in differences in the observed emission properties such as the radio spectrum, the surface brightness, and the spectral index profiles (see e.g. the work of Donnert et al. 2016).

Several observations have challenged the DSA mechanism. These observations have revealed that for some relics the derived X-ray Mach numbers are low, namely weak shocks with $M \leq 3$ (Brunetti \& Jones 2014; van Weeren et al. 2019). For such weak shocks, the DSA mechanism is not efficient enough to accelerate particles up to $\mathrm{GeV}$ energies from the thermal pool (see also Botteon et al. 2020). Only recently, a new class of radio relics with low surface brightness, and emissivity compatible with the standard DSA scenario might 
have been discovered at low frequency using the Low Frequency Array (LOFAR, see Locatelli et al. 2020). In some cases, the Mach number inferred from X-ray observations and the one obtained from radio spectra are not in agreement (see e.g. van Weeren et al. 2016). In addition, some authors have reported the presence of a break in the spectrum, based on interferomteric observations, which is inconsistent with DSA (Trasatti et al. 2015; Stroe et al. 2016).

All these findings motivates the search for complementary/alternative models with respect to the DSA to explain the observed emission. In particular, it has been proposed that nearby active galactic nuclei can inject cosmic ray electrons (Enßlin \& Gopal-Krishna 2001), a scenario confirmed in some cases (Bonafede et al. 2014; van Weeren et al. 2017; Botteon et al. 2019), or that the injection is caused by powerful galactic wind (Völk \& Atoyan 1999). Spherically expanding shocks with fossil particle populations (Kang \& Ryu 2015) re-accelerated through DSA can generated curved spectra, while a non-uniform magnetic field in the relic region (Donnert et al. 2016) could cause a steepening in the radio spectrum. Multiple shocks along the line of sight (Hong, Kang \& Ryu 2015, and reference therein) can explain the inconsistency between X-ray and radio derived Mach numbers. However, the modelling proposed by Hong et al. (2015) and Donnert et al. (2016) in the framework of the DSA does not solve the low efficiency problem which makes the acceleration of particles from the thermal pool unrealistic.

The inconsistency between the X-ray and radio derived Mach numbers (see e.g. van Weeren et al. 2010; Akamatsu et al. 2015, for radio and X-ray derived Mach number), and the steepening in the flux density spectrum (Stroe et al. 2016) was also observed for the northern relic of the galaxy cluster CIZA J2242.8+5301. This cluster (redshift $z=0.1921$ ) was discovered in the X-rays by Kocevski et al. (2007), while its diffuse radio sources, a central radio halo and a pair of opposite radio relics, were discovered by van Weeren et al. (2010). Its northern relic is one of the most famous and extensively studied radio relics (van Weeren et al. 2010; Stroe et al. 2013, 2014, 2016; Loi et al. 2017; Hoang et al. 2017; Kierdorf et al. 2017; Di Gennaro et al. 2018) and it is often considered a textbook example of radio relics because of its $\sim 2 \mathrm{Mpc}$ arc-shaped morphology and uniform brightness along its length.

Interferometric measurements of the northern relic at 15.85 and $30 \mathrm{GHz}$ (Stroe et al. 2016) obtained with the Arcminute Microkelvin Imager (AMI, Zwart et al. 2008) and Combined Array for Research in Millimeter-wave Astronomy (CARMA, Scott et al. 2004) telescopes, triggered the search for alternative physics, as they indicated a steepening of the spectrum which is not compatible with the standard DSA model. Nevertheless, it should be noted that, especially at high frequency, interferometric observations can suffer from the missing zero baseline problem. While single-dish telescopes can retain angular structures as large as the observed area, interferometers can detect a maximum angular structure corresponding to their minimum baseline, since their minimum baseline is not equal to zero as in the case of single dish. This means that interferometric observations are not able to fully recover the flux density of very extended sources. In particular, AMI small array (SA) and large array (LA) observations at $15.85 \mathrm{GHz}$ have a corresponding large angular scale $\theta_{\text {LAS }} \sim 11$ and $\sim 2$ arcmin, respectively. CARMA at $30 \mathrm{GHz}$ was blind to scales above $\theta_{\text {LAS }} \sim 3.5 \mathrm{arcmin}$. The northern relic covers an angular scale larger than 11 arcmin. Therefore, even if Stroe et al. (2016) tried to correct for this effect, obtaining reliable flux density measurements from these telescopes is an hard task.

Kierdorf et al. (2017) excluded a possible steepening between $153 \mathrm{MHz}$ and $8.35 \mathrm{GHz}$ and model the radio relic with a power law with a spectral index $\alpha \sim 0.9$ while DSA predicts $\alpha>1$.
In a previous work (Loi et al. 2017), we established, using data between $\sim 300 \mathrm{MHz}$ and $\sim 8 \mathrm{GHz}$ that the CIZA J2242.8+5301 northern relic spectral behaviour was consistent with the DSA model in this frequency range assuming a continuous injection of relativistic particles. From the spectral modelling, we also derived a Mach number consistent within the errors with the X-ray estimate (Akamatsu et al. 2015). However, the uncertainty about the relic behaviour at higher frequency remained.

In this work, we show the results of a large observing program (code: 72-19, PI: Francesca Loi) conducted at the Sardinia Radio Telescope facility (SRT, Bolli et al. 2015; Prandoni et al. 2017) aiming at observing this famous relic at $18.6 \mathrm{GHz}$ with the 7 -feed $K$-band receiver of this single-dish telescope in full-Stokes mode. We also present $14.25 \mathrm{GHz}$ data acquired with the Effelsberg single-dish telescope (code: 73-19, PI: Rainer Beck).

In Section 2, we describe the observational set up, the data reduction, and the imaging procedure for both total intensity and polarized intensity images. In Section 3, we show the resulting total intensity image and the measurement of the relic flux, and compare these value with literature data. In Section 4, we discuss the SunyaevZel'dovich effect (SZ, Sunyaev \& Zeldovich 1972, 1980) which could affect our measurements and based on the expected/observed contamination we give a rough estimate of the magnetic field in the relic region. In Section 5, we show the polarized intensity image and we discuss the polarimetric properties of the detected sources. In Section 6, we discuss our findings and draw the conclusions.

Throughout this paper, we assume a $\Lambda$-cold dark matter cosmology with $H_{0}=71 \mathrm{~km} \mathrm{~s}^{-1} \mathrm{Mpc}^{-1}, \Omega_{\mathrm{m}}=0.27$, and $\Omega_{\Lambda}=0.73$. At the redshift of CIZA J2242.8+5301 $(z=0.1921), 1$ arcmin corresponds to $189.9 \mathrm{kpc}$.

\section{OBSERVATIONS AND DATA REDUCTION}

\subsection{SRT observations}

We used the 7-feed $K$-band receiver of the SRT to observe at a central frequency of $18.6 \mathrm{GHz}$ inside a bandwidth of $1200 \mathrm{MHz}$. These data were acquired with the SArdinia Roach2-based Digital Architecture for Radio Astronomy back end (Sardara, Melis et al. 2018) using $1500 \mathrm{MHz}$ bandwidth and 1024 channels of $1.46 \mathrm{MHz}$ width in full-Stokes mode.

The observations have been carried out between 2020 January and April, for a total of $240 \mathrm{~h}$ divided in 27 slots of about 6-12 h each. A summary of the observing program is reported in Table 1. During each slot, we observed the primary calibrator 3C 286 (or $3 \mathrm{C} 147$ if the former was not available) to calibrate bandpass and flux density scale. We performed sky dips to derive the trend of the system temperature with elevation during our observations. We then modelled the $T_{\text {sys }}$ trend with the airmass model to obtain the zenithal opacity, $\tau$. The values of $\tau$ derived from the sky dip were generally in good agreement with those provided by the radiometer working at the SRT site (Buffa et al. 2017). We also used the calibrator 3C 286 as a reference for the absolute polarization position angle (we assumed the values from Perley \& Butler 2013). If 3C 286 was not available, we used 3C 138 instead. The sources 3C 84 and NGC 7027 , that we considered to be completely unpolarized, were used to correct for the on-axis instrumental polarization. The northern relic of CIZA J2242.8+5301 was observed with the on-the-fly strategy in the equatorial frame, covering an area of $21 \times 15 \mathrm{arcmin}^{2}$ centred on

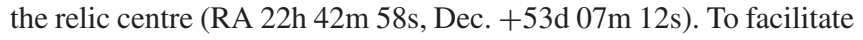
the removal of the scan noise, we acquired orthogonal on-the-fly maps along the RA and Dec. directions. Individual subscans within 
Table 1. Details about the SRT observations.

\begin{tabular}{lccc}
\hline & $\begin{array}{c}\text { Obs. time } \\
\text { (h) }\end{array}$ & Calibrators & Number of maps \\
\hline 12 Jan 2020 & 8 & 3C 286, 3C 84 & 8 RA + 8 Dec. \\
24 Jan 2020 & 7 & 3C 286, 3C 84 & 11 RA + 10 Dec. \\
01 Feb 2020 & 8 & 3C 286, 3C 84 & 10 RA + 10 Dec. \\
04 Feb 2020 & 10 & 3C 286, 3C 84 & 11 RA + 11 Dec. \\
06 Feb 2020 & 8 & 3C 147, 3C 84, 3C 138 & 8 RA + 8 Dec. \\
07 Feb 2020 & 8 & 3C 286, 3C 84 & 11 RA + 11 Dec. \\
08 Feb 2020 & 8 & 3C 286, 3C 84 & 8 RA + 8 Dec. \\
09 Feb 2020 & 8 & 3C 286, 3C 84 & 11 RA + 11 Dec. \\
11 Feb 2020 & 11 & 3C 147, 3C 84, 3C 138 & 6 RA + 6 Dec. \\
12 Feb 2020 & 8 & 3C 286, 3C 84 & 10 RA + 10 Dec. \\
26 Feb 2020 & 11 & 3C 286, 3C 84 & 13 RA + 13 Dec. \\
27 Feb 2020 & 13 & 3C 286, 3C 84 & 17 RA + 17 Dec. \\
28 Feb 2020 & 13 & 3C 286, 3C 84 & 17 RA + 17 Dec. \\
29 Feb 2020 & 7 & 3C 286, NGC 7027 & 10 RA + 10 Dec. \\
10 Mar 2020 & 6 & 3C 286, 3C 84 & 9 RA + 9 Dec. \\
14 Mar 2020 & 8 & 3C 286, 3C 84 & 12 RA + 12 Dec. \\
15 Mar 2020 & 8 & 3C 286, 3C 84 & 11 RA + 11 Dec. \\
19 Mar 2020 & 10 & 3C 147, 3C 84, 3C 138 & 14 RA + 14 Dec. \\
24 Mar 2020 & 9 & 3C 147, 3C 84, 3C 138 & 11 RA + 11 Dec. \\
25 Mar 2020 & 6 & 3C 48, 3C 147 & 8 RA + 8 Dec. \\
26 Mar 2020 & 7 & 3C 48, 3C 84, 3C 138 & 9 RA + 9 Dec. \\
02 Apr 2020 & 8 & 3C 286, 3C 84 & 11 RA + 11 Dec. \\
03 Apr 2020 & 6 & 3C 48, 3C 84, 3C 138 & 5 RA + 5 Dec. \\
04 Apr 2020 & 10 & 3C 286, 3C 84 & 16 RA + 16 Dec. \\
10 Apr 2020 & 8 & 3C 286, 3C 84 & 12 RA + 14 Dec. \\
14 Apr 2020 & 11 & 3C 147, 3C 84, 3C 138 & 11 RA + 11 Dec. \\
16 Apr 2020 & 10 & 3C 286, 3C 84 & 14 RA + 14 Dec. \\
\hline & & &
\end{tabular}

these maps are separated by 15 arcsec, in order to sample the FWHM (full width at half-maximum) of the SRT beam with about 4 pixels along each direction.

We performed the data reduction and the imaging with the proprietary software package Single-dish Spectral-polarimetry Software (SCUBE, Murgia et al. 2016). We used a standardized pipeline for the calibration and imaging: we excised all the RFIs at well-known specific frequencies and we applied an automatic flagging procedure to remove the remaining RFIs. We then determined the opacity, we subtracted the calibrator baselines with a linear fit of each scan based on the first and last 10 per cent of the data, we determined the bandpass solution, we gridded the calibrators in a regular grid with a pixel size of 15 arcsec, and we used the resulting images to fit a 2D-Gaussian to determine the flux scale and the leakage terms.

Our primary goal is to image the emission of the relic and the point sources in the field of view of target. Since we are uninterested to retain any large-scale foreground emission, in the data of the target we removed the baseline scan-by-scan by fitting a second-order polynomial to the 'cold-sky' regions devoid of both discrete sources and of the galaxy cluster extended emission (relics and halo). These cold-sky regions are identified using a mask created with the $1.4 \mathrm{GHz}$ SRT+WSRT image presented in Loi et al. (2017), convolved with a beam FWHM of 1 arcmin. In this way, we removed the baselevel related to the receiver noise, the atmospheric emission, and the largescale foreground sky emission. We then imaged the spectral cubes using a regular grid of 15 arcsec of pixel size and we averaged all the spectral channel to increase the signal-to-noise ratio. The images from all the observing slots are stacked together to reduce the noise level. We stack the RA and Dec. scans by mixing their stationary wavelet transform (SWT) coefficients (see Murgia et al. 2016). The de-stripping resulting from the SWT stacking is effective in isolating and removing the noise along the scan direction. After the first stack was completed, we returned to the individual images using the higher signal-to-noise image as a reference model to flag all residual low-level RFIs or small-scale atmospheric fluctuations that were not captured at the flag and baseline removal stages. This refined flagging step significantly improved the quality of the images. In order to verify the consistency of the flux density scale calibration between all the different observing slots, we performed a self-calibration procedure using the cluster central source (RA 22h $42 \mathrm{~m} \mathrm{51.30s,}$ Dec. $+53 \mathrm{~d} 04 \mathrm{~m} 41.40 \mathrm{~s}$ ) as reference. We assumed that this source has a stable flux density of $8.8 \mathrm{mJy}$ and, by mean of a 2D Gaussian fit to this source, we calculated a correction factor for each observing slot. By analysing the results of the self-calibration procedure, we deduced that the rms scatter of the flux density calibration scale through our project is of about $\sim 10$ per cent. This scatter could be assumed as an estimate for our systematic uncertainty of the flux density calibration.

The polarized image has been obtained after correcting for the leakage term determined with 3C 84 (or NGC 7027). We calibrated the polarized angle and fraction with 3C 286 (or 3C 138). To perform the imaging of the $Q$ and $U$ Stokes parameters, we followed the same steps described above for the total intensity. The images of the polarized intensity $P$ and the polarization angle $\Psi$ have been derived from the Stokes parameters solving for the following equations:

$P=\sqrt{Q^{2}+U^{2}}$

$\Psi=0.5 \cdot \arctan \frac{U}{Q}$.

We also corrected the polarization images for the Rician bias (see Murgia et al. 2016).

The maximum rotation measure (RM) observed for this relic (see Loi et al. 2017; Kierdorf et al. 2017) is $\mathrm{RM}=-400 \mathrm{rad} \mathrm{m}^{-2}$. At $18.6 \mathrm{GHz}$ this would cause a rotation of the polarization plane of $\Delta \Psi \sim 6 \mathrm{deg}$. Therefore, it is unlikely that our signal can undergo significant depolarization effect but it is indeed very close to the intrinsic polarization properties of the relic.

\subsection{Effelsberg observations}

We also observed the northern relic of CIZA J2242.8+5301 with the new two-horn Ku-band receiver of the Effelsberg 100-m telescope that provides two channels of $2.5 \mathrm{GHz}$ bandwidth each, centred on 14.25 and $16.75 \mathrm{GHz}$. The system temperatures are 33 and $44 \mathrm{~K}$. In 2019 December and 2020 January, we obtained 12 coverages of a field of $12 \times 9 \operatorname{arcmin}^{2}$ by scanning ('on-the fly') in alternating RA and Dec. directions. The total on-source observation time was $7 \mathrm{~h}$. Data processing (RFI removal, base-level corrections, and combination of the coverages in RA and Dec. with the 'basket weaving' technique) was performed with the NOD3 software package (Müller et al. 2017) using 3C 147 or 3C 48 as flux density calibrators. The final image at $14.25 \mathrm{GHz}$ with a resolution of $49 \operatorname{arcsec}$ has a rms noise of $1 \mathrm{mJy} \mathrm{beam}^{-1}$. To increase the signal-to-noise ratio, we smoothed the image to 72 arcsec.

In the second channel centred at $16.75 \mathrm{GHz}$ no significant signal could be detected. The second horn is separated in azimuthal direction by 3.85 arcmin and can be used to reduce weather effects. As this requires scanning larger areas in azimuthal direction and the weather was excellent, this method was not used. The linear polarization signal was also recorded, but was too weak due to the relatively short observation time. 


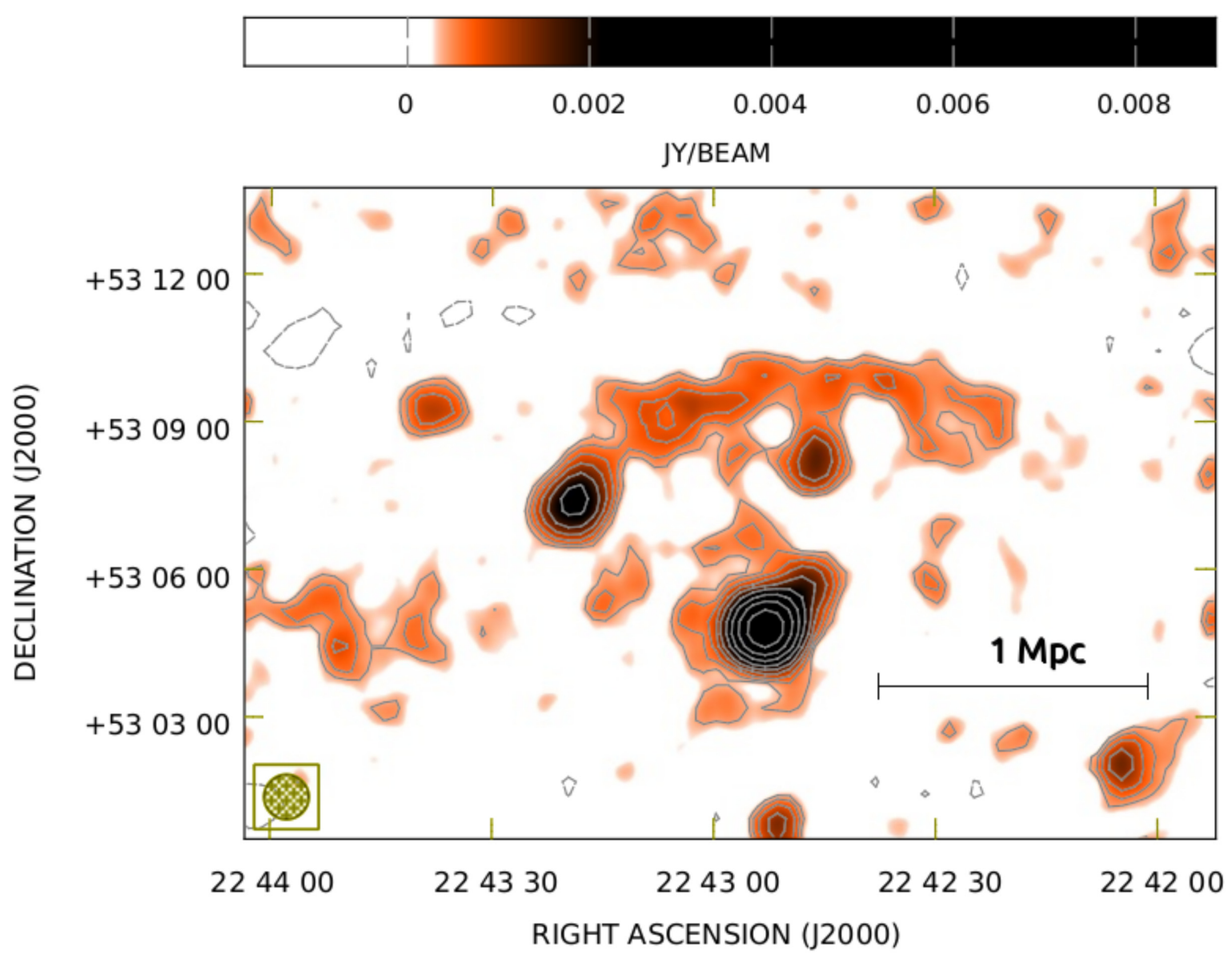

Figure 1. $18.6 \mathrm{GHz}$ SRT total intensity image between 18 and $19.2 \mathrm{GHz}$. Contours start at $3 \sigma$ where $\sigma=0.13 \mathrm{mJy}_{\text {beam }}^{-1}$ and increment with a $\sqrt{2}$ factor. Dashed contours are negative contours drawn at $-3 \sigma$. The beam size is shown in the bottom left corner of the image, its FWHM corresponds to $\sim 0.9$ arcmin.

\section{TOTAL INTENSITY RESULTS AND ANALYSIS}

\subsection{SRT image and analysis}

Fig. 1 shows the resulting $18.6 \mathrm{GHz}$ SRT image obtaining by averaging the data between 18 and $19.2 \mathrm{GHz}$. The noise is $0.13 \mathrm{mJy} \mathrm{beam}^{-1}$, and the beam size is 0.9 arcmin. Solid contours are drawn from $3 \sigma$ increasing by a factor of $\sqrt{2}$, while dashed contours are the negative $-3 \sigma$ contours.

We clearly detected the emission associated to the brightest radio sources in the field including the northern relic which extends over a length of $\sim 1.8 \mathrm{Mpc}$ with a deconvolved width ranging from $\sim 40$ up to $\sim 160 \mathrm{kpc}$. The relic surface brightness is not homogeneous across the arc, remarking the filamentary structure observed for the first time at 1.5 and $3 \mathrm{GHz}$ (Di Gennaro et al. 2018).

In the central part of the image, we can observe the two radio galaxies (labelled D and E in fig. 7 of Loi et al. 2017) which are unresolved at our beam resolution. A patch of radio emission is located eastwards from the centre. We noticed that this structure does not correspond to the candidate relic observed at lower frequencies (see Hoang et al. 2017) and that it can be seen only in the LOFAR $145 \mathrm{MHz}$ low-resolution (i.e. $35 \mathrm{arcsec}$ ) image.

Fig. 2 shows the positive contours of Fig. 1 overlaid on the WSRT+SRT $1.4 \mathrm{GHz}$ total intensity image reported in Loi et al. (2017). A grey arrow indicates the eastern structure mentioned above.

Fig. 2 also shows a blue contour, traced following the $3 \sigma$ contours of the $18.6 \mathrm{GHz}$ image, which indicates the northern relic area.

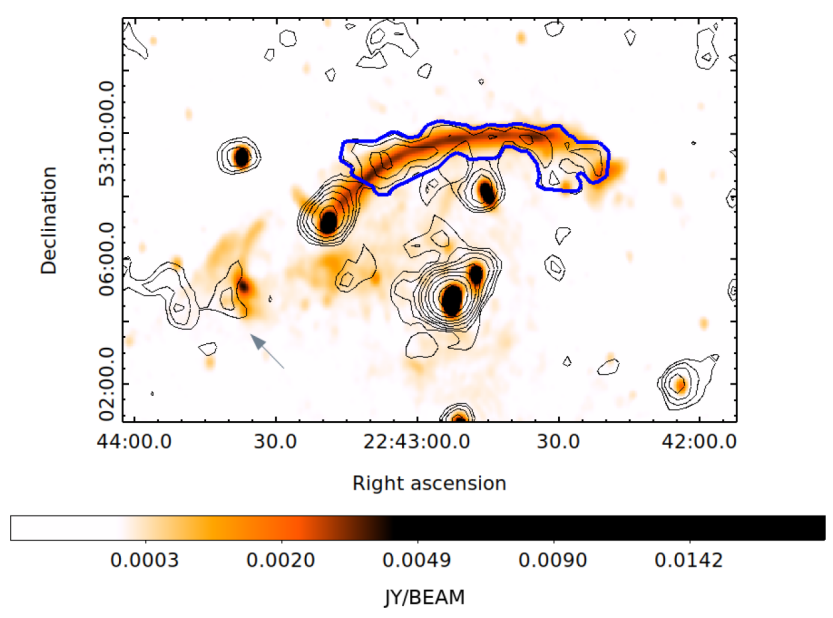

Figure 2. $1.4 \mathrm{GHz}$ WSRT+SRT total intensity image from Loi et al. (2017) with the positive contours of Fig. 1. The blue contour indicates the region where we estimated the flux density of the relic.

We measured the flux density of the relic inside this region which covers an area of 13 beam areas. Similar to what has been done in Kierdorf et al. (2017), using the same region, we evaluated the residual base level of the image by computing the mean of the surface brightness in 10 different regions of the image with no obvious source. The resulting mean surface brightness computed 


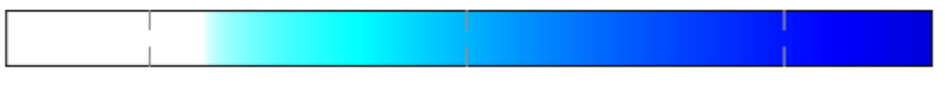

0

5

10

mJY/BEAM

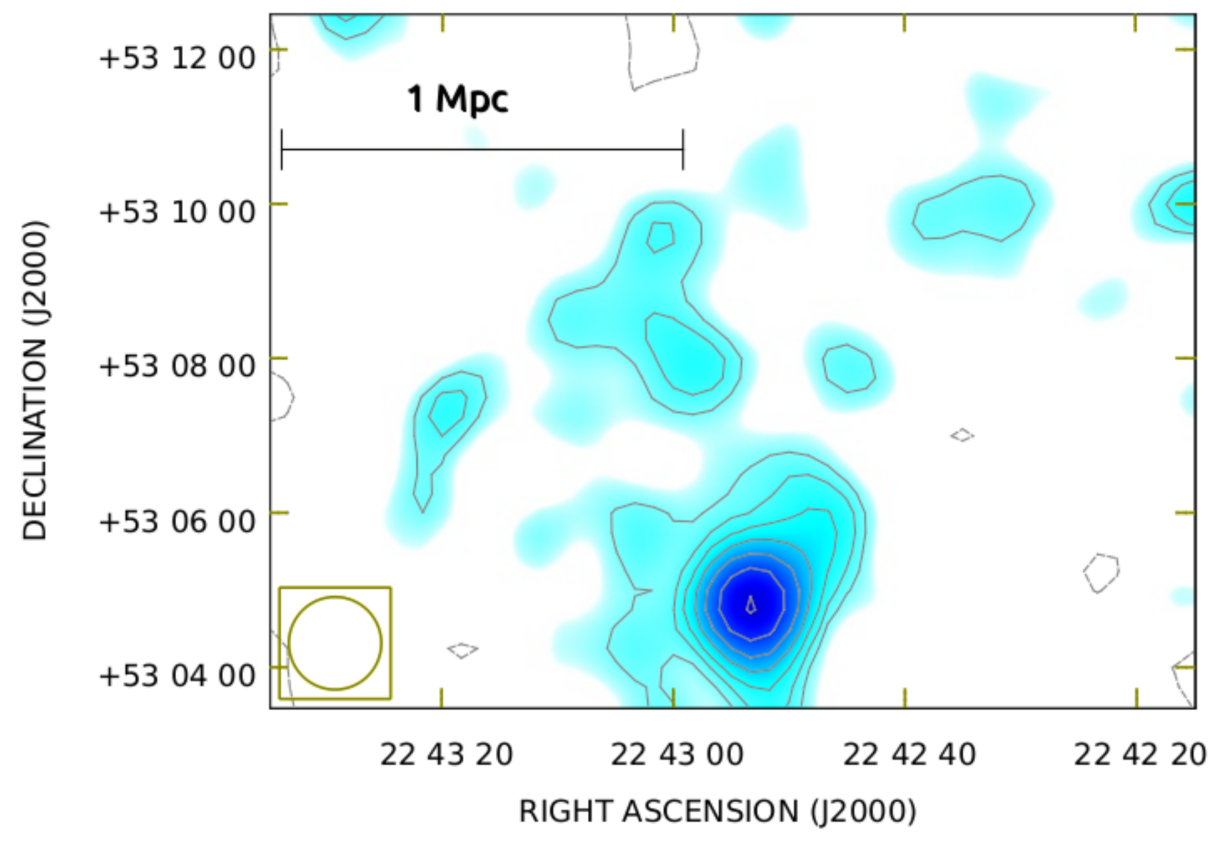

Figure 3. $14.25 \mathrm{GHz}$ Effelsberg total intensity image between 13.0 and $15.5 \mathrm{GHz}$. Positive contours (solid line) start at $3 \sigma$ where $\sigma=0.5 \mathrm{mJy}$ beam ${ }^{-1}$ and increase with a factor of $\sqrt{2}$, while negative contours at shown at $-3 \sigma$ (dashed line). The beam size is shown in the bottom left corner, its FWHM corresponds to 72 arcsec.

from these 10 regions is $\sim 4.5 \mu \mathrm{Jy}_{\text {beam }}{ }^{-1}$ and the associated $\mathrm{rms}$ is $\sim 75 \mu \mathrm{Jy}_{\text {beam }}^{-1}$. The base level correction factor of the image is given by this mean multiplied by the number of beams of the relic region, and corresponds to $58.5 \mu \mathrm{Jy}$. We compute the error on the flux density as:

$\Delta S_{v}=\sqrt{\left(f \cdot S_{v}\right)^{2}+\sigma^{2} \cdot N_{\mathrm{BEAM}}+(\Delta \mathrm{BL})^{2}}$,

where $f$ is the systematic flux uncertainty that we assume to be equal to 10 per cent, $\sigma$ is the noise image, and $N_{\text {BEAM }}$ is the number of beam corresponding to the relic area. $\triangle \mathrm{BL}$ is the error associated to the base level correction which is equal to the rms of the regions divided by the square root of $N_{\mathrm{BEAM}}$.

The flux density of the relic at $18.6 \mathrm{GHz}$ corrected for the base level is:

$S_{18.6 \mathrm{GHz}}=(7.67 \pm 0.90) \mathrm{mJy}$.

\subsection{Effelsberg image and analysis}

Fig. 3 shows the $14.25 \mathrm{GHz}$ Effelsberg image, convolved at a resolution of $72 \mathrm{arcsec}$, shown in the bottom left corner. The noise in this image is $0.5 \mathrm{mJy}^{\text {beam }}{ }^{-1}$. Solid contours are drawn from $3 \sigma$ increasing by a factor of $\sqrt{2}$, while dashed contours are the negative $-3 \sigma$ contours.

In this image, we detected the central sources of the cluster, namely the $\mathrm{D}$ and $\mathrm{E}$ sources, and patches associated with the northern radio relic of CIZA J2242.8+5301.

Using the same blue region of Fig. 2, we evaluated the relic flux in the $14.25 \mathrm{GHz}$ Effelsberg image at its original resolution of 49 arcsec.
At $14.25 \mathrm{GHz}$, the relic flux is:

$S_{14.25 \mathrm{GHz}}=(9.5 \pm 3.9) \mathrm{mJy}$.

\subsection{Spectral fitting}

Fig. 4 shows the flux density of the relic as a function of frequency. We included our 14.25 and $18.6 \mathrm{GHz}$ measurements as a green and red dot, respectively to the most updated results in the literature (Kierdorf et al. 2017; Loi et al. 2017; Hoang et al. 2017; Di Gennaro et al. 2018) shown as black dots. More details about the measurements are in Table 2. These data have been modelled with a power-law spectrum which resulted to be the best-fitting model, with a reduced chi-square $\chi_{v}^{2}=0.26$. In the fitting procedure, we did not include the interferometric measurements at 16 and $30 \mathrm{GHz}$ by Stroe et al. (2013) because we suspected that a significant fraction of the flux density could be missed in these estimates due to the lack of sensitivity at scales larger than their minimum baseline.

We measured again the flux at $6.6 \mathrm{GHz}$ considering now a $3 \sigma$ threshold instead of $5 \sigma$ (see Loi et al. 2017, for the details about this measurement). The resulting integrated spectral index calculated between $145 \mathrm{MHz}$ and $18.6 \mathrm{GHz}$ is:

$\alpha=1.12 \pm 0.03$,

and confirms what found in recent works over a smaller frequency range (i.e. between $145 \mathrm{MHz}$ and $2.2 \mathrm{GHz}$, and between 1.5 and $3 \mathrm{GHz}$ as reported by Hoang et al. 2017; Di Gennaro et al. 2018, respectively). Our measurements exclude a possible steepening of the relic spectrum up to a frequency of $19 \mathrm{GHz}$. 


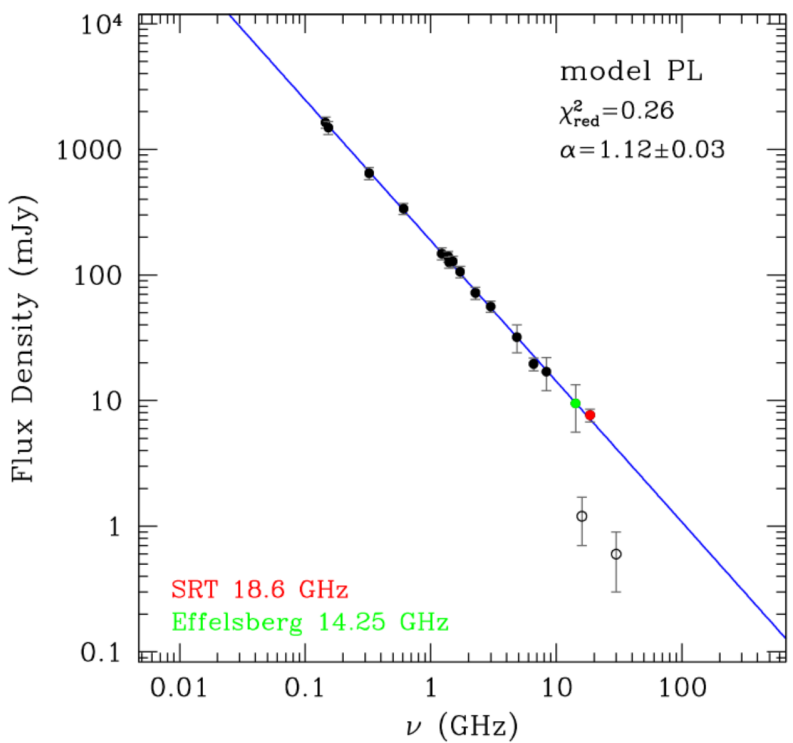

Figure 4. The CIZA J2242.8+5301 northern relic flux density as a function of frequency. Black dots are measurements from the literature, while the green and red dots are the 14.251 and $18.6 \mathrm{GHz}$ measurement presented in this work. The power-law best fit is shown as a solid blue line. The two empty points are measurements from Stroe et al. (2016) which we did not include in the fitting procedure.

Table 2. Flux density measurements of the CIZA J2242.8+5301 northern relic shown in Fig. 4. The measurement at $6.6 \mathrm{GHz}$ has been repeated considering a $3 \sigma$ threshold.

\begin{tabular}{lcc}
\hline $\begin{array}{l}\text { Frequency } \\
(\mathrm{GHz})\end{array}$ & $S_{v}(\mathrm{mJy})$ & Reference \\
\hline 0.145 & $1637 \pm 168$ & Hoang et al. (2017) \\
0.153 & $1488 \pm 171$ & Hoang et al. (2017) \\
0.323 & $646 \pm 71$ & Hoang et al. (2017) \\
0.608 & $337 \pm 35$ & Hoang et al. (2017) \\
1.221 & $148 \pm 16$ & Hoang et al. (2017) \\
1.382 & $140 \pm 14$ & Hoang et al. (2017) \\
1.4 & $126 \pm 12.6$ & Loi et al. $(2017)$ \\
1.5 & $128.1 \pm 12.81$ & Di Gennaro et al. $(2018)$ \\
1.714 & $106 \pm 11$ & Hoang et al. $(2017)$ \\
2.272 & $72 \pm 8$ & Hoang et al. $(2017)$ \\
3 & $56.1 \pm 5.61$ & Di Gennaro et al. $(2018)$ \\
4.85 & $32 \pm 8$ & Kierdorf et al. (2017) \\
6.6 & $19.6 \pm 2.3$ & Loi et al. $(2017)$ \\
8.35 & $17 \pm 5$ & Kierdorf et al. $(2017)$ \\
14.25 & $9.5 \pm 3.9$ & This work \\
16 & $1.2 \pm 0.5$ & Stroe et al. $(2016)$ \\
18.6 & $7.67 \pm 0.90$ & This work \\
30 & $0.6 \pm 0.3$ & Stroe et al. (2016) \\
\hline
\end{tabular}

\section{SUNYAEV-ZEL'DOVICH DECREMENT}

Observations at high-frequency (i.e. $>10 \mathrm{GHz}$ ) can be affected by the SZ effect which consists in a inverse-Compton interaction between cosmic microwave background (CMB) photons and thermal ICM particles. As a result, the synchrotron emission associated to cluster-embedded sources is reduced from true values because the background CMB emission is shifted towards higher frequencies (see Birkinshaw 1999) adding a 'negative' contamination to the flux density of cluster-embedded sources.
We can reasonably assert that the measurements presented in this work are not affected by the large-scale radial SZ decrement since such an effect would be mitigated in the baseline subtraction procedure. Nevertheless, as described by Basu et al. (2016), a sharp pressure jump due to a shock is expected to generate a 'localized' SZ small-scale decrement at the observed frequencies and it is reasonable that our images are affected by this contamination (even if we remark that in the case of the northern relic of CIZA J2242.8+5301, the surface brightness and pressure jump were not clearly detected, but see Ogrean et al. 2014).

Even if we do not have a direct measurement of the SZ at the position of the shock, we could try to estimate the expected decrement at $18.6 \mathrm{GHz}$ from numerical simulation. According to Basu et al. (2016) the Comptonization parameter $y$ at the relic location exceeds by $-1.4 \times 10^{4}$ with respect to the radial $y$ trend which we assume to have been absorbed by the baseline subtraction. Using equation (3) by Basu et al. (2016) to compute the expected negative surface brightness associated to the CMB inverse-Compton emission, after multiplying this quantity by the number of beam area covered by the relic to derive the flux density $S_{\mathrm{SZ}}$, we can compute the SZ decrement at $18.6 \mathrm{GHz}$ as follows:

$\Delta S_{\mathrm{SZ}}=-2$ y $S_{\mathrm{SZ}}=-0.9 \mathrm{mJy}$.

This value corresponds to the uncertainty associated with our measurement. At $14.25 \mathrm{GHz}$ the decrement is equal to $-0.3 \mathrm{mJy}$. This means that it is very hard to detect a SZ decrement in our images and in fact we do not see any evident deviation from the power-law trend in the relic flux density spectrum. We can fix the -11.7 per cent (which is $\Delta S_{\mathrm{SZ}}$ at $18.6 \mathrm{GHz}$ divided by the relic flux density) as an upper limit of the contamination due to the SZ effect, assuming that no other effects are contributing to compensate it. Considering equation (15) by Basu et al. (2016) reported below:

$$
\begin{aligned}
\left(\frac{S_{v}^{\text {relic }}}{\Delta S_{\mathrm{SZ}}}\right) \simeq & -9 \times 10^{4} \cdot\left(\frac{\xi_{e / p}}{0.05}\right) \cdot\left(\frac{M}{3}\right) \\
& \cdot\left(\frac{T_{u}}{1 \mathrm{keV}}\right)^{1 / 2} \cdot\left(\frac{W}{100 \mathrm{kpc}}\right)^{-1} \cdot(1+z)^{-(4+\delta / 2)} \\
& \cdot \frac{B_{\text {relic }}^{1+\delta / 2}}{B_{\mathrm{CMB}}^{2}+B_{\text {relic }}^{2}} \cdot\left(\frac{v}{1.4 \mathrm{GHz}}\right)^{-(2+\delta / 2)},
\end{aligned}
$$

we can then tentatively investigate how the magnetic field in the relic region $B_{\text {relic }}$ changes with the Mach number $M$ assuming the previous SZ decrement upper limit. In the above formula, $S_{v}^{\text {relic }} / \Delta S_{\mathrm{SZ}}$ is the SZ decrement in percentage fixed to -11.7 per cent, $\xi_{e / p}$ is the ratio between relativistic protons and electron that we fix equal to 0.0026 according to Kang, Ryu \& Jones (2012), $T_{u}$ is the upstream temperature equal to $2.7 \mathrm{keV}$ (Akamatsu et al. 2015), $W$ is the relic width that is equal to $\sim 100 \mathrm{kpc}$ (see Section 3 ), $\delta$ is the spectral index of the relativistic particle distribution assumed to be equal to 4.2 (Kang et al. 2012), and $B_{\mathrm{CMB}}=3.24(1+\mathrm{z})^{2} \mu \mathrm{G}$. We stress that many approximations have been done to derive this formula, for instance it is assumed that the relic is perfectly seen edge-on. However, this simplified formula can give us an alternative way to estimate of the magnetic field strength in the relic region. The blue line in Fig. 5 shows the relic magnetic field values at different Mach number at $v=18.6 \mathrm{GHz}$. This has been derived computing the resulting Mach number values at different magnetic field. We draw dotted vertical lines at the X-ray and radio derived Mach number. To these values corresponds a magnetic field strength of $B_{\text {relic }} \sim 3-4 \mu \mathrm{G}$, which is an intermediate value between what found by van Weeren et al. (2010), $B_{\text {relic }} \sim 5-7 \mu \mathrm{G}$, and Kierdorf et al. (2017), $B_{\text {relic }} \sim 2.4 \mu \mathrm{G}$, by 


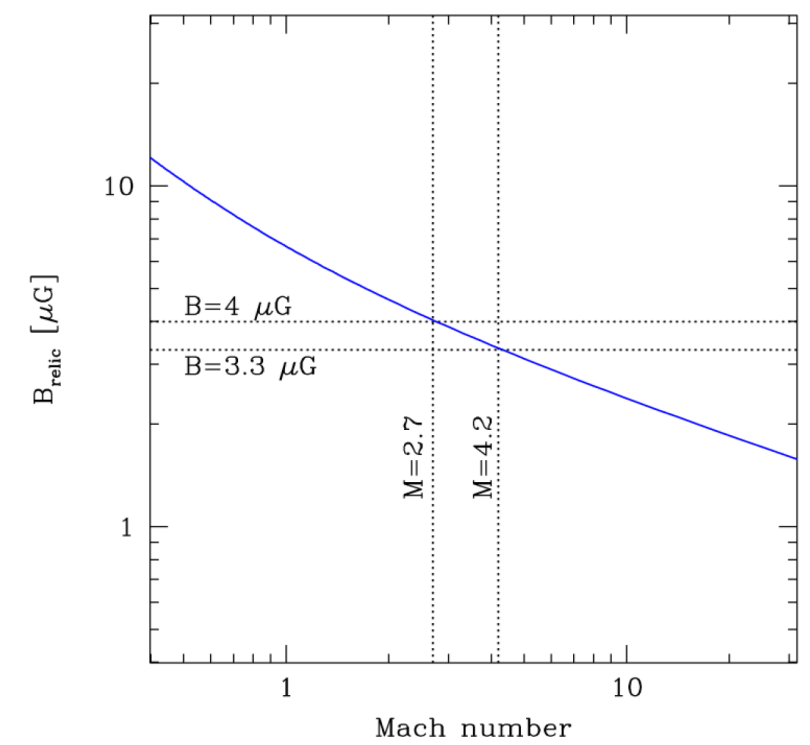

Figure 5. Magnetic field strength versus Mach number. See equation (8).

fitting the radial profile of the relic and with equipartition arguments respectively.

\section{POLARIZATION PROPERTIES AT 18.6 GHZ}

Fig. 6 shows the polarized intensity associated with the northern and central part of the galaxy cluster CIZA J2242.8+5301 detected at $18.6 \mathrm{GHz}$ with the SRT. This is the first polarized image of a radio relic at these frequencies and it is fundamental to evaluate its intrinsic polarization properties which are strongly related to the local magnetic field. Contours are the total intensity contours already shown in Fig. 1. The length and the orientation of the overlaid vectors represent the intensity and the orientation of the radio wave electric field vectors. We draw them considering all pixels with a total intensity larger than $3 \sigma$, which also have a fractional linear polarization $\mathrm{S} / \mathrm{N} \geq 2$ and errors on the polarization angle smaller than $15^{\circ}$.

The E-field vectors are aligned perpendicular to the relic filaments. This has already been observed at other frequencies, namely at $4.9 \mathrm{GHz}$ (van Weeren et al. 2010), at $6.6 \mathrm{GHz}$ (Loi et al. 2017), at 4.85 and $8.35 \mathrm{GHz}$ (Kierdorf et al. 2017), and it is expected in presence of a shock wave. Visually, only the magnetic field lines at $90^{\circ}$ from the line of sight are observed, while we are blind to the other magnetic fields components, since the resulting E-vectors are not propagating towards us. The 'observable' magnetic fields lines are aligned with the shock surface and the E-vectors are perpendicular to it. We note that, if the magnetic field would already have been aligned on large scales, its non-zero components would be perpendicular to the shock direction, generating E-vectors similar to what observed in the previous case. Therefore, we cannot distinguish between a largescale ordered magnetic field and a turbulent magnetic field structure in the presence of a shock wave which compress the magnetic field in a thin layer.

Fig. 7 shows the polarization fraction computed in nine boxes with size corresponding to the beam size located from east to west across the relic. The average polarization fraction at this resolution is equal to $(47 \pm 13)$ per cent. We detected the polarized signal associated to the D and E sources at the centre of the cluster which shows a mean polarization fraction of $\sim 12$ per cent. The eastern structure is strongly polarized, with an average polarization fraction of $\sim 70$ per cent.

\section{DISCUSSION AND CONCLUSIONS}

The northern relic of CIZA J2242.8+5301 is a privileged site to study the acceleration of relativistic electrons by merger shocks in the ICM. Even if it is one of the most cited radio relics, it has posed a lot of questions since its discovery, and it has challenged the standard model of shock acceleration in cluster outskirts. With this work, we have shown that the radio relic spectral behaviour is well modelled with a power law from $145 \mathrm{MHz}$ up to $19 \mathrm{GHz}$. The measurements presented in this work constitute clear evidence that there is no steepening at high frequencies at variance with earlier claims (Stroe et al. 2016). The inconsistency between this measurement and the $16 \mathrm{GHz}$ and $30 \mathrm{GHz}$ taken with the AMI and CARMA interferometers is mostly likely due to the lack of sensitivity of these interferometers on scales larger than their minimum baseline. It is clear that interferometers can miss flux associated with sources extended on large angular scales and therefore might not be sufficient in the study of extended sources, making a combination with single-dish observations essential.

We know that in the context of the DSA (Hoeft \& Brüggen 2007), from the fitted integrated spectral index, we can derive the Mach number as:

$M=\sqrt{\frac{\alpha+1}{\alpha-1}}$.

According to what found in this work, the Mach number should be $M=4.2_{-0.6}^{+0.4}$. This value is significantly different from what derived with X-ray observations $\left(M_{\mathrm{X}}=2.7_{-0.4}^{+0.7}\right.$, Akamatsu et al. 2015). However, as also previous works highlighted (Hoang et al. 2017; Di Gennaro et al. 2018), deriving the Mach number from the integrated spectral index can lead to significant errors in some cases. Indeed, the previous formula has been derived under the assumption that the properties of the shock and the downstream gas remain constant during the electron cooling. Recent cosmological simulations of radio relics by Wittor et al. (2019) have shown that both the Mach number and the magnetic field are not uniform across the shock front. Furthermore, they showed that the downstream magnetic field is by far not constant. By comparing spectral index measurements from observations and simulation, Rajpurohit et al. (2020) argued that the spectral index is most-likely biased by the high value tail of the underlying Mach number distribution. A better way to estimate the Mach number is to evaluate it from the injected spectral index, measuring this quantity in the injection region from images at very high-resolution or from the colour-colour diagram in case of projection effect.

Following Ensslin et al. (1998), we can use our polarized image to investigate the possible projection of the relic in the framework of the DSA.

Under the assumption of a weak magnetic field in the relic region, that is if the magnetic pressure is lower than the gas pressure, the average fractional linear polarization $<\Pi>$ is a function of the viewing angle $\theta$ as:

$$
<\Pi_{\text {weak }}>=\frac{3 \gamma+3}{3 \gamma+7} \frac{\sin ^{2} \theta}{\frac{2 \mathrm{R}^{2}}{\mathrm{R}^{2}-1}-\sin ^{2} \theta},
$$

where $R$ is the compression ratio defined as:

$R=\frac{\alpha+1}{\alpha-\frac{1}{2}}$, 

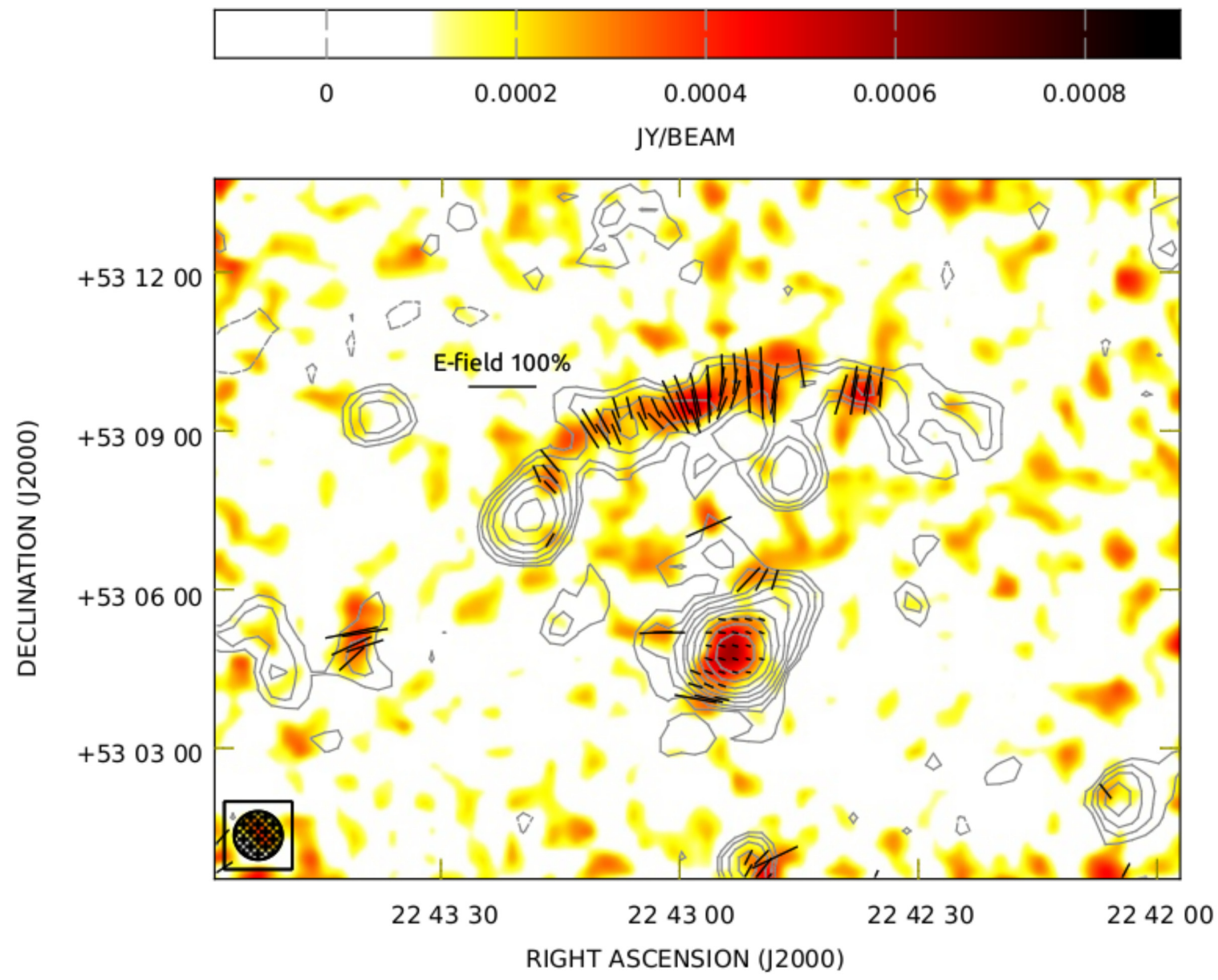

Figure 6. $18.6 \mathrm{GHz}$ SRT polarized intensity image between 18 and $19.2 \mathrm{GHz}$. Contours are the same as the total intensity contours shown in Fig. 1 . Vectors represent the intensity and the orientation of the E-field. They have been traced for pixels with a total intensity larger than $3 \sigma$, a fractional linear polarization $\mathrm{S} / \mathrm{N} \geq 2$, and an error on the polarization angle smaller than $15^{\circ}$.

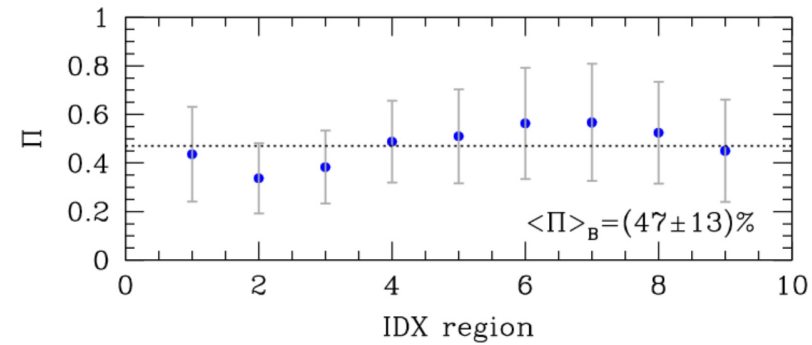

Figure 7. Polarization fraction computed in boxes with size corresponding to the beam size. The dashed line shows the mean value reported in the bottom left corner of the plot.

and $\gamma$ is the spectral index of the electron power-law like energy distribution, with $\gamma=2 \alpha+1$. On the other side, if the magnetic field pressure is higher that the gas pressure, we have:

$$
<\Pi_{\text {strong }}>=\frac{3 \gamma+3}{3 \gamma+7} \frac{\sin ^{2} \theta}{\frac{2}{15} \frac{13 \mathrm{R}-7}{\mathrm{R}-1}-\sin ^{2} \theta} .
$$

The scalar mean $\langle\Pi>$ of the fractional polarization is evaluated from the $Q, U$, and $I$ Stokes parameters averaged over the entire relic area. If the shock relic is seen edge-on, that is forming a viewing angle of $90^{\circ}$, then we observe its maximum averaged polarization fraction, since the polarized signal is due to the amplified magnetic field vector components along the shock direction. For a relic seen face-on, the magnetic field vector components which are illuminated by the relic relativistic particles are not aligned, therefore we observe a null averaged polarization fraction. This is shown in Fig. 8, where we plot the polarization fraction as a function of the viewing angle in the case of weak (blue) and strong (red) magnetic fields, assuming $\alpha=1.12$.

We measured the scalar mean of the polarization fraction which is equal to $\langle\Pi>=(42 \pm 17)$ per cent. This value is slightly different from what reported in the previous section since there we measured the mean polarization fraction from values obtained within beam size regions, while here we computed the mean from $Q, U$, and $I$ averaged over the entire relic region. Using the geometrical argument of Ensslin et al. (1998), we computed a viewing angle of $33^{\circ}$ which means that the shock direction is forming an angle of $57^{\circ}$ with respect to the plane of the sky. A black dot shows this estimate in Fig. 8. It is clear that the assumption of a spherical geometry of the shock wave is a simplification of the real shock geometry. For this reason is not surprising that the black dot is far from the plotted curves. Nevertheless, according to the measured value of polarization fraction we can see that the radio relic is not perfectly seen edge-on, but we are observing it from a viewing angle between $45^{\circ}$ and $80^{\circ}$, 


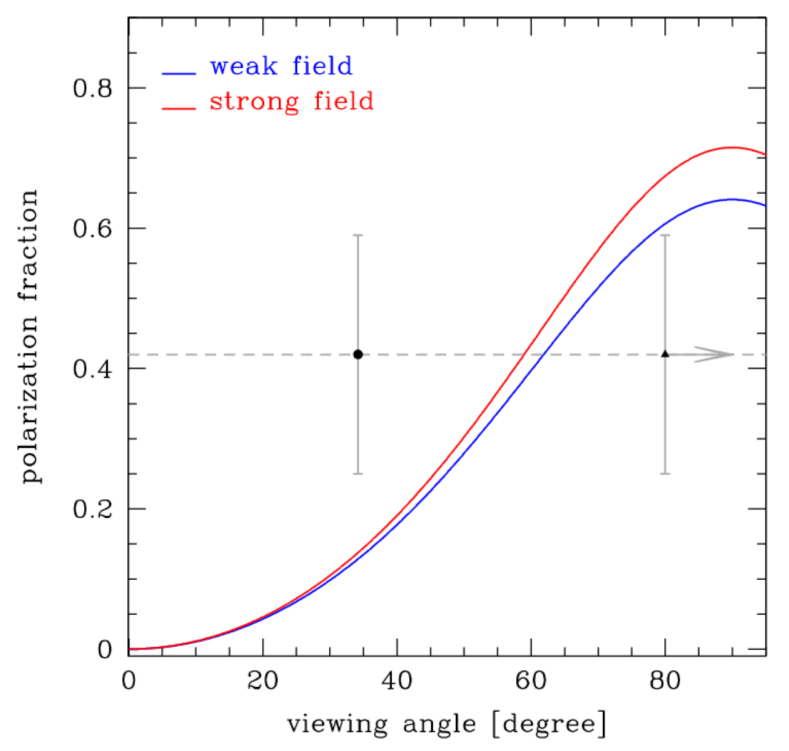

Figure 8. Polarization fraction as a function of the viewing angle in the case of weak (blue) and strong (red) magnetic fields.

that is the relic could be inclined with respect to the plane of the sky by an angle ranging from $10^{\circ}$ to $45^{\circ}$. Numerical simulation of this relic (van Weeren et al. 2011) determined that the shock direction is inclined with respect to the plane of the sky by an angle $\lesssim 10^{\circ}$. This estimates is shown as a black triangle in the plot.

The interpretation of the mean polarized fraction in the framework of the DSA confirms that the relic is not seen perfectly edge-on and can explain the inconsistency between X-ray and radio derived Mach number. In addition, it is important to mention that X-ray observations do not clearly confirm the presence of a shock wave, since the shock has been only observed as a jump in temperature but not in the surface brightness ( $<2 \sigma$ detection, see Ogrean et al. 2014). Shock waves with Mach number $M \sim 3$ should show a significant jump in the X-ray surface brightness profile.

Another question which needs to be answered is what is the source of cosmic ray electrons. As already pointed out in the Introduction, shock waves with weak Mach number cannot efficiently accelerate particles from the thermal pool (Brunetti \& Jones 2014; van Weeren et al. 2019). Therefore, also for this particular case, we expect that a pre-existing relativistic particle population has been re-accelerated through the DSA mechanism at the shock passage. Due to the large extension of the relic, it is unlikely that the two nearby radio galaxies can supply such relativistic electrons. Nevertheless, fossil particles, injected by galaxies which are no longer active, may have accumulated in the relic area during the past history of the cluster. A multiple shock (Hong et al. 2015) structure along the line of sight could be the responsible of the (re-)acceleration of such fossil particles in the northern relic of CIZA J2242.8+5301. This would also explain the inconsistency between radio and X-rayderived Mach numbers. Interestingly, Ogrean et al. (2014) identified additional inner small density discontinuities both on and off the merger axis with Chandra data, which could be interpreted, as suggested by the authors, as shock fronts.

To summarize, we presented new measurements of the northern radio relic of CIZA $\mathbf{J} 2242.8+5301$ obtained with the Effelsberg and the SRT single-dish telescopes. We found a flux density of $S_{14.25 \mathrm{GHz}}=(9.5 \pm 3.9) \mathrm{mJy}$ and $S_{18.6 \mathrm{GHz}}=(7.67 \pm 0.90) \mathrm{mJy}$ at 14.25 GHz with the Effelsberg and $18.6 \mathrm{GHz}$ with the SRT tele- scopes, respectively. The best-fitting modelling of the radio relic spectrum between $143 \mathrm{MHz}$ and $19 \mathrm{GHz}$ is a power law with spectral index $\alpha=(1.12 \pm 0.03)$. Our measurements exclude a possible steepening of the relic spectrum up to a frequency of $19 \mathrm{GHz}$. We estimated the possible SZ contamination and we determined that the expected decrement both at 14.25 and $18.6 \mathrm{GHz}$ would be within the uncertainty associated with our measurements. Assuming the modelling of Basu et al. (2016), we also inferred a rough estimate of the magnetic field in the relic region based on the derived SZ decrement upper limit at $18.6 \mathrm{GHz}$ and this resulted to be $B_{\text {relic }} \sim 3-4 \mu \mathrm{G}$, a value close to what found in previous works with different approaches (van Weeren et al. 2010; Kierdorf et al. 2017). For the first time, we also detected the polarized intensity associated to the relic at $18.6 \mathrm{GHz}$. The mean polarization fraction calculated in boxes of the same beam size of the image is equal to $(47 \pm 13)$ per cent while the scalar mean computed over the entire relic area, dividing the mean polarized intensity by the mean total intensity, is equal to $(44 \pm 18)$ per cent. In the last paragraphs, we speculated about the origin of this radio relic. We suggested that this relic emission could be due to a fossil plasma re-accelerated by a multiple shock structure which is propagating in a direction inclined with respect to the plane of the sky. High-resolution and high-frequency polarized radio images as well as deep X-ray images could help to constrain the viewing angle and the shock structure of the relic respectively, allowing us to validate the proposed scenario.

\section{ACKNOWLEDGEMENTS}

We thank the anonymous referee for the useful suggestions and comments which help to improve our paper. We thank Sorina Reile for her work on reducing the Effelsberg data. FL and PS acknowledge financial support from the Italian Minister for Research and Education (MIUR), project FARE, project code R16PR59747, project name FORNAX-B. DW is funded by the Deutsche Forschungsgemeinschaft (DFG, German Research Foundation) - 441694982. AB acknowledges financial support from the MIUR, project FARE. AB e MB acknowledges financial support from the ERC-Stg DRANOEL, no. 714245. DW, KR, and FV acknowledge financial support from the ERC Starting Grant 'MAGCOW', no. 714196. The SRT (Bolli et al. 2015; Prandoni et al. 2017) is funded by the Ministry of Education, University and Research (MIUR), Italian Space Agency (ASI), the Autonomous Region of Sardinia (RAS), and INAF itself and is operated as National Facility by the National Institute for Astrophysics (INAF). The development of the SARDARA back-end has been funded by the RAS using resources from the Regional Law 7/2007 'Promotion of the scientific research and technological innovation in Sardinia' in the context of the research project CRP49231 (year 2011, PI: Possenti): 'High resolution sampling of the Universe in the radio band: an unprecedented instrument to understand the fundamental laws of the nature'. Partly based on observations with the 100-m telescope of the MPIfR (Max-PlanckInstitut für Radioastronomie) at Effelsberg.

\section{DATA AVAILABILITY}

The data underlying this article will be shared on reasonable request to the corresponding author.

\section{REFERENCES}

Akamatsu H. et al., 2015, A\&A, 582, A87

Basu K., Vazza F., Erler J., Sommer M., 2016, A\&A, 591, A142 
Birkinshaw M., 1999, Phys. Rev., 310, 97

Blandford R. D., Ostriker J. P., 1978, ApJ, 221, L29

Bolli P. et al., 2015, J. Astron. Instrum., 4, 1550008-880

Bonafede A. et al., 2014, ApJ, 785, 1

Botteon A. et al., 2019, A\&A, 622, A19

Botteon A., Brunetti G., Ryu D., Roh S., 2020, A\&A, 634, A64

Brunetti G., Jones T. W., 2014, Int. J. Mod. Phys. D, 23, 1430007

Buffa F., Bolli P., Sanna G., Serra G., 2017, MeScT, 28, 014004

Di Gennaro G. et al., 2018, ApJ, 865, 24

Donnert J. M. F., Stroe A., Brunetti G., Hoang D., Roettgering H., 2016, MNRAS, 462, 2014

Drury L. O., 1983, RPPh, 46, 973

Ensslin T. A., Biermann P. L., Klein U., Kohle S., 1998, A\&A, 332, 395

Enßlin T. A., Gopal-Krishna, 2001, A\&A, 366, 26

Hoang D. N. et al., 2017, MNRAS, 471, 1107

Hoeft M., Brüggen M., 2007, MNRAS, 375, 77

Hong S. E., Kang H., Ryu D., 2015, ApJ, 812, 49

Kang H., Ryu D., 2015, ApJ, 809, 186

Kang H., Ryu D., Jones T. W., 2012, ApJ, 756, 97

Kierdorf M., Beck R., Hoeft M., Klein U., van Weeren R. J., Forman W. R., Jones C., 2017, A\&A, 600, A18

Kocevski D. D., Ebeling H., Mullis C. R., Tully R. B., 2007, ApJ, 662, 224

Locatelli N. T. et al., 2020, MNRAS, 496, L48

Loi F. et al., 2017, MNRAS, 472, 3605

Melis A. et al., 2018, J. Astron. Instrum., 7, 1850004

Müller P., Krause M., Beck R., Schmidt P., 2017, A\&A, 606, A41

Murgia M. et al., 2016, MNRAS, 461, 3516

Ogrean G. A., Brüggen M., van Weeren R., Röttgering H., Simionescu A., Hoeft M., Croston J. H., 2014, MNRAS, 440, 3416
Perley R. A., Butler B. J., 2013, ApJS, 206, 16

Prandoni I. et al., 2017, A\&A, 608, A40

Rajpurohit K. et al., 2020, A\&A, 636, A30

Scott S. L. et al., 2004, in Ochsenbein F., Allen M. G., Egret D., eds, ASP Conf. Ser. Vol. 314, Astronomical Data Analysis Software and Systems (ADASS) XIII. Astron. Soc. Pac., San Francisco, p. 768

Stroe A. et al., 2016, MNRAS, 455, 2402

Stroe A., van Weeren R. J., Intema H. T., Röttgering H. J. A., Brüggen M., Hoeft M., 2013, A\&A, 555, A110

Stroe A., Harwood J. J., Hardcastle M. J., Röttgering H. J. A., 2014, MNRAS, 445,1213

Sunyaev R. A., Zeldovich Y. B., 1972, Comment Astrophys. Space Phys., 4, 173

Sunyaev R. A., Zeldovich I. B., 1980, MNRAS, 190, 413

Trasatti M. et al., 2015, A\&A, 575, A45

van Weeren R. J. et al., 2016, ApJ, 818, 204

van Weeren R. J. et al., 2017, Nat. Astron., 1, 0005

van Weeren R. J. et al., 2019, Space Sci. Rev., 215, 16

van Weeren R. J., Röttgering H. J. A., Brüggen M., Hoeft M., 2010, Science, 330,347

van Weeren R. J., Brüggen M., Röttgering H. J. A., Hoeft M., 2011, MNRAS, 418,230

Völk H. J., Atoyan A. M., 1999, APh, 11, 73

Wittor D., Hoeft M., Vazza F., Brüggen M., Domínguez-Fernández P., 2019, MNRAS, 490, 3987

Zwart J. T. L. et al., 2008, MNRAS, 391, 1545

This paper has been typeset from a $\mathrm{T}_{\mathrm{E}} \mathrm{X} / \mathrm{L} \mathrm{A} \mathrm{E} \mathrm{X}$ file prepared by the author. 


\section{List of astronomical key words (Updated on 2020 January)}

This list is common to Monthly Notices of the Royal Astronomical Society, Astronomy and Astrophysics, and The Astrophysical Journal. In order to ease the search, the key words are subdivided into broad categories. No more than six subcategories altogether should be listed for a paper.

The subcategories in boldface containing the word 'individual' are intended for use with specific astronomical objects; these should never be used alone, but always in combination with the most common names for the astronomical objects in question. Note that each object counts as one subcategory within the allowed limit of six.

The parts of the key words in italics are for reference only and should be omitted when the keywords are entered on the manuscript.

\section{General}

editorials, notices

errata, addenda

extraterrestrial intelligence

history and philosophy of astronomy

miscellaneous

obituaries, biographies

publications, bibliography

sociology of astronomy

standards

\section{Physical data and processes}

acceleration of particles

accretion, accretion discs

asteroseismology

astrobiology

astrochemistry

astroparticle physics

atomic data

atomic processes

black hole physics

chaos

conduction

convection

dense matter

diffusion

dynamo

elementary particles

equation of state

gravitation

gravitational lensing: micro

gravitational lensing: strong

gravitational lensing: weak

gravitational waves

hydrodynamics

instabilities

line: formation

line: identification

line: profiles

magnetic fields

magnetic reconnection

(magnetohydrodynamics) MHD

masers

molecular data

molecular processes

neutrinos

nuclear reactions, nucleosynthesis, abundances

opacity

plasmas

polarization radiation: dynamics

radiation mechanisms:general

radiation mechanisms: non-thermal

radiation mechanisms: thermal

radiative transfer

relativistic processes

scattering

shock waves

solid state: refractory

solid state: volatile

turbulence

waves

\author{
Astronomical instrumentation, methods and techniques \\ atmospheric effects \\ balloons \\ instrumentation: adaptive optics \\ instrumentation: detectors \\ instrumentation: high angular resolution \\ instrumentation: interferometers \\ instrumentation: miscellaneous \\ instrumentation: photometers \\ instrumentation: polarimeters \\ instrumentation: spectrographs \\ light pollution \\ methods: analytical \\ methods: data analysis \\ methods: laboratory: atomic \\ methods: laboratory: molecular \\ methods: laboratory: solid state \\ methods: miscellaneous \\ methods: numerical \\ methods: observational \\ methods: statistical \\ site testing \\ space vehicles \\ space vehicles: instruments \\ techniques: high angular resolution \\ techniques: image processing \\ techniques: imaging spectroscopy \\ techniques: interferometric \\ techniques: miscellaneous \\ techniques: photometric \\ techniques: polarimetric \\ techniques: radar astronomy \\ techniques: radial velocities \\ techniques: spectroscopic \\ telescopes
}




\section{Astronomical data bases}

astronomical data bases: miscellaneous

atlases

catalogues

surveys

virtual observatory tools

\section{Software}

software: data analysis

software: development

software: documentation

software: public release

software: simulations

\author{
Astrometry and celestial mechanics \\ astrometry \\ celestial mechanics \\ eclipses \\ ephemerides \\ occultations \\ parallaxes \\ proper motions \\ reference systems \\ time
}

\section{The Sun}

Sun: abundances

Sun: activity

Sun: atmosphere

Sun: chromosphere

Sun: corona

Sun: coronal mass ejections (CMEs)

Sun: evolution

Sun: faculae, plages

Sun: filaments, prominences

Sun: flares

Sun: fundamental parameters

Sun: general

Sun: granulation

Sun: helioseismology

Sun: heliosphere

Sun: infrared

Sun: interior

Sun: magnetic fields

Sun: oscillations

Sun: particle emission

Sun: photosphere

Sun: radio radiation

Sun: rotation

(Sun:) solar-terrestrial relations

(Sun:) solar wind

(Sun:) sunspots

Sun: transition region

Sun: UV radiation

Sun: X-rays, gamma-rays

\section{Planetary systems}

comets: general

comets: individual: ... .

Earth

interplanetary medium

Kuiper belt: general

Kuiper belt objects: individual: . . . meteorites, meteors, meteoroids minor planets, asteroids: general

minor planets, asteroids: individual: ...

Moon

Oort Cloud

planets and satellites: atmospheres

planets and satellites: aurorae

planets and satellites: composition

planets and satellites: detection

planets and satellites: dynamical evolution and stability

planets and satellites: formation

planets and satellites: fundamental parameters

planets and satellites: gaseous planets

planets and satellites: general

planets and satellites: individual: ...

planets and satellites: interiors

planets and satellites: magnetic fields

planets and satellites: oceans

planets and satellites: physical evolution

planets and satellites: rings

planets and satellites: surfaces

planets and satellites: tectonics

planets and satellites: terrestrial planets

planet-disc interactions

planet-star interactions

protoplanetary discs

zodiacal dust

Stars

stars: abundances

stars: activity

stars: AGB and post-AGB

stars: atmospheres

(stars:) binaries (including multiple): close

(stars:) binaries: eclipsing

(stars:) binaries: general

(stars:) binaries: spectroscopic

(stars:) binaries: symbiotic

(stars:) binaries: visual

stars: black holes

(stars:) blue stragglers

(stars:) brown dwarfs

stars: carbon

stars: chemically peculiar

stars: chromospheres

(stars:) circumstellar matter

stars: coronae

stars: distances

stars: dwarf novae

stars: early-type

stars: emission-line, Be

stars: evolution

stars: flare

stars: formation

stars: fundamental parameters

(stars:) gamma-ray burst: general

(stars:) gamma-ray burst: individual: ...

stars: general

(stars:) Hertzsprung-Russell and colour-magnitude

diagrams

stars: horizontal branch

stars: imaging

stars: individual: ...

stars: interiors 
stars: jets

stars: kinematics and dynamics

stars: late-type

stars: low-mass

stars: luminosity function, mass function

stars: magnetars

stars: magnetic field

stars: massive

stars: mass-loss

stars: neutron

(stars:) novae, cataclysmic variables

stars: oscillations (including pulsations)

stars: peculiar (except chemically peculiar)

(stars:) planetary systems

stars: Population II

stars: Population III

stars: pre-main-sequence

stars: protostars

(stars:) pulsars: general

(stars:) pulsars: individual: ...

stars: rotation

stars: solar-type

(stars:) starspots

stars: statistics

(stars:) subdwarfs

(stars:) supergiants

(stars:) supernovae: general

(stars:) supernovae: individual: ...

stars: variables: Cepheids

stars: variables: Scuti

stars: variables: general

stars: variables: RR Lyrae

stars: variables: S Doradus

stars: variables: T Tauri, Herbig Ae/Be

(stars:) white dwarfs

stars: winds, outflows

stars: Wolf-Rayet

Interstellar medium (ISM), nebulae

ISM: abundances

ISM: atoms

ISM: bubbles

ISM: clouds

(ISM:) cosmic rays

(ISM:) dust, extinction

ISM: evolution

ISM: general

(ISM:) HII regions

(ISM:) Herbig-Haro objects

ISM: individual objects: . . .

(except planetary nebulae)

ISM: jets and outflows

ISM: kinematics and dynamics

ISM: lines and bands

ISM: magnetic fields

ISM: molecules

(ISM:) photodissociation region (PDR)

(ISM:) planetary nebulae: general

(ISM:) planetary nebulae: individual: ...

ISM: structure

ISM: supernova remnants
The Galaxy

Galaxy: abundances

Galaxy: bulge

Galaxy: centre

Galaxy: disc

Galaxy: evolution

Galaxy: formation

Galaxy: fundamental parameters

Galaxy: general

(Galaxy:) globular clusters: general

(Galaxy:) globular clusters: individual: ...

Galaxy: halo

Galaxy: kinematics and dynamics

(Galaxy:) local interstellar matter

Galaxy: nucleus

(Galaxy:) open clusters and associations: general

(Galaxy:) open clusters and associations: individual: ...

(Galaxy:) solar neighbourhood

Galaxy: stellar content

Galaxy: structure

\section{Galaxies}

galaxies: abundances

galaxies: active

galaxies: bar

(galaxies:) BL Lacertae objects: general

(galaxies:) BL Lacertae objects: individual: ...

galaxies: bulges

galaxies: clusters: general

galaxies: clusters: individual: ... .

galaxies: clusters: intracluster medium

galaxies: disc

galaxies: distances and redshifts

galaxies: dwarf

galaxies: elliptical and lenticular, cD

galaxies: evolution

galaxies: formation

galaxies: fundamental parameters

galaxies: general

galaxies: groups: general

galaxies: groups: individual: ...

galaxies: haloes

galaxies: high-redshift

galaxies: individual: . . .

galaxies: interactions

(galaxies:) intergalactic medium

galaxies: irregular

galaxies: ISM

galaxies: jets

galaxies: kinematics and dynamics

(galaxies:) Local Group

galaxies: luminosity function, mass function

(galaxies:) Magellanic Clouds

galaxies: magnetic fields

galaxies: nuclei

galaxies: peculiar

galaxies: photometry

(galaxies:) quasars: absorption lines

(galaxies:) quasars: emission lines

(galaxies:) quasars: general 
(galaxies:) quasars: individual: ... .

(galaxies:) quasars: supermassive black holes galaxies: Seyfert

galaxies: spiral

galaxies: starburst

galaxies: star clusters: general

galaxies: star clusters: individual: . . .

galaxies: star formation

galaxies: statistics

galaxies: stellar content

galaxies: structure

\section{Cosmology}

(cosmology:) cosmic background radiation

(cosmology:) cosmological parameters

(cosmology:) dark ages, reionization, first stars

(cosmology:) dark energy

(cosmology:) dark matter

(cosmology:) diffuse radiation

(cosmology:) distance scale

(cosmology:) early Universe

(cosmology:) inflation

(cosmology:) large-scale structure of Universe

cosmology: miscellaneous

cosmology: observations

(cosmology:) primordial nucleosynthesis

cosmology: theory

\section{Resolved and unresolved sources as a function of wavelength}

gamma-rays: diffuse background

gamma-rays: galaxies

gamma-rays: galaxies: clusters

gamma-rays: general

gamma-rays: ISM

gamma-rays: stars

infrared: diffuse background

infrared: galaxies

infrared: general

infrared: ISM

infrared: planetary systems

infrared: stars

radio continuum: galaxies

radio continuum: general

radio continuum: ISM

radio continuum: planetary systems

radio continuum: stars

radio continuum: transients

radio lines: galaxies

radio lines: general

radio lines: ISM

radio lines: planetary systems

radio lines: stars

submillimetre: diffuse background

submillimetre: galaxies

submillimetre: general

submillimetre: ISM

submillimetre: planetary systems

submillimetre: stars

ultraviolet: galaxies ultraviolet: general

ultraviolet: ISM

ultraviolet: planetary systems

ultraviolet: stars

X-rays: binaries

$\mathrm{X}$-rays: bursts

X-rays: diffuse background

X-rays: galaxies

$\mathrm{X}$-rays: galaxies: clusters

$\mathrm{X}$-rays: general

X-rays: individual: ... .

X-rays: ISM

X-rays: stars

\section{Transients}

(transients:) black hole mergers

(transients:) black hole - neutron star mergers

(transients:) fast radio bursts

(transients:) gamma-ray bursts

(transients:) neutron star mergers

transients: novae

transients: supernovae

transients: tidal disruption events 